\title{
An improved static corrugation model
}

Cite as: J. Chem. Phys. 149, 234702 (2018); https://doi.org/10.1063/1.5058271

Submitted: 18 September 2018 . Accepted: 20 November 2018 . Published Online: 20 December 2018

P. Spiering $(\mathbb{D})$ M. Wijzenbroek, and M. F. Somers
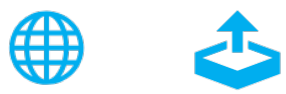

Export Citation

\section{ARTICLES YOU MAY BE INTERESTED IN}

Communication: Becke's virial exciton model gives accurate charge-transfer excitation energies

The Journal of Chemical Physics 149, 231101 (2018); https://doi.org/10.1063/1.5078515

Towards the description of adsorption of water in slit-like pores with walls covered by molecular brushes

The Journal of Chemical Physics 149, 234703 (2018); https://doi.org/10.1063/1.5066552

Lattice models and Monte Carlo methods for simulating DNA origami self-assembly

The Journal of Chemical Physics 149, 234905 (2018); https://doi.org/10.1063/1.5051835 


\title{
An improved static corrugation model
}

\author{
P. Spiering, ${ }^{1}$ M. Wijzenbroek ${ }^{2}$ and M. F. Somers ${ }^{1, a)}$ \\ ${ }^{1}$ Leiden University, Leiden, Zuid-holland 2300 RA, The Netherlands \\ ${ }^{2}$ University of Amsterdam, Amsterdam, The Netherlands
}

(Received 18 September 2018; accepted 20 November 2018; published online 20 December 2018)

\begin{abstract}
Accurately describing surface temperature effects for the dissociation of $\mathrm{H}_{2}$ on $\mathrm{Cu}(111)$ remains challenging. While Ab initio Molecular Dynamics (AIMD), the current state-of-the-art method for modelling such systems, can produce accurate results, it is computationally very expensive to use for extensive testing of, for example, density functionals. A chemically accurate static corrugation model for $\mathrm{H}_{2}$ and $\mathrm{D}_{2}$ on $\mathrm{Cu}(111)$ dissociation was made by introducing effective three-body interactions as well as an $\mathrm{H}_{2}$-bond dependence and fitting the model to density functional theory energies for 15113 different configurations. Reaction probabilities and rovibrational (in)elastic scattering probabilities were computed and compared to experiments and other calculations. Theoretical and experimental results are in good agreement, except for the reaction of $(\mathrm{v}=0, \mathrm{~J}=0) \mathrm{H}_{2}$ where both AIMD and the newly developed static corrugation model, both based on the same underlying density functional, predict a similar deviation from the experiment. Published by AIP Publishing. https://doi.org/10.1063/1.5058271
\end{abstract}

\section{INTRODUCTION}

Heterogeneously catalysed processes such as the HaberBosch ${ }^{1}$ process and the hydrogen and oxygen evolution reactions of water splitting ${ }^{2}$ are essential for modern day industry. To gain insight into how these processes are catalysed efficiently, for example, by metal interfaces, the reaction mechanism is broken down into elementary reaction steps, which are subsequently studied individually. Understanding these elementary reaction steps can then, hopefully, lead to better catalysis of chemical processes on surfaces. One such elementary reaction step is the dissociation of $\mathrm{H}_{2}$ and $\mathrm{D}_{2}$ on $\mathrm{Cu}(111)$, which has been extensively studied both experimentally ${ }^{3-8}$ and theoretically. ${ }^{9-16}$

For modeling such systems, the Born-Oppenheimer approximation (BOA) is often used to separate the nuclear and electron dynamics. A ground state electronic structure theory such as density functional theory (DFT) is then used to compute the energy at several nuclear configurations resulting in a potential energy surface (PES). For the dissociative chemisorption probabilities of $\mathrm{H}_{2}$ and $\mathrm{D}_{2}$ on $\mathrm{Cu}(111)$, it is known that the BOA performs well. ${ }^{17,18}$ One way to determine the overall accuracy of the electronic structure is to calculate the probability of $\mathrm{H}_{2}$ reacting on the $\mathrm{Cu}(111)$ surface at specific translational and rovibrational energies and compare the result with experiments. DFT can be used together with the specific reaction parameter (SRP) method to compute a chemically accurate PES for the $\mathrm{H}_{2}$ on the $\mathrm{Cu}(111)$ system. ${ }^{11}$

In practice, chemical processes at surfaces are performed at high surface temperatures, which complicates the

a)Electronic mail: m.somers@chem.leidenuniv.nl fundamental understanding even further. To include the effect of surface temperature on the dissociation of $\mathrm{H}_{2}$ and $\mathrm{D}_{2}$ on $\mathrm{Cu}(111)$ however, the PES must somehow take surface displacements into account. It is computationally convenient to reduce the PES to a $6 \mathrm{D} \mathrm{PES}{ }^{14,15}$ and describe the effect of surface displacements as a perturbation of the 6D PES. This was done previously with the static corrugation model (SCM) ${ }^{13}$ Here it was assumed that $\mathrm{H}_{2}$ interacts with essentially a static snapshot of a thermally equilibrated $\mathrm{Cu}(111)$ surface. This was motivated for $\mathrm{H}_{2}$ and $\mathrm{D}_{2}$ on $\mathrm{Cu}(111)$ due to the large mass mismatch allowing no significant energy exchange due to collisions of the molecule with the surface. Furthermore, the comparatively slow velocity of thermally equilibrated $\mathrm{Cu}$ atoms results in no significant surface motion during the short interaction time of $\mathrm{H}_{2}$ at the $\mathrm{Cu}(111)$ surface. In contrast, it is also possible to compute the electronic structure on an "as needed" basis using ab initio molecular dynamics (AIMD), ${ }^{9}$ circumventing the need to make any further approximations, albeit at increased computational effort. The SCM was able to reproduce experimental and AIMD molecular beam experiments ${ }^{9}$ within chemical accuracy $(1 \mathrm{kcal} / \mathrm{mol})$, even though the results of the fitting procedure used suggested that the errors should be larger. Recently, it has also become possible to use high-dimensional neural network potentials ${ }^{19-21}$ (HD-NNPs) to describe the effect of surface displacements in molecule surface reactions. While this seems a promising alternative to both AIMD and the SCM, we are not aware of any accurate $\mathrm{HD}-\mathrm{NNP}$ for $\mathrm{H}_{2}$ on $\mathrm{Cu}(111)$, using the same DFT functional as was used for AIMD,${ }^{9}$ at the moment of writing.

Our goal is now to improve the SCM for the $\mathrm{H}_{2}$ and $\mathrm{D}_{2}$ on the $\mathrm{Cu}(111)$ system to even more accurately reproduce the results of AIMD and experimental data, while maintaining the advantage in computational effort. 


\section{METHODS}

\section{A. $A b$ Initio calculations for $\mathrm{H}_{2}$ at $\mathrm{Cu}(111)$}

For the $\mathrm{H}_{2}$ on the $\mathrm{Cu}(111)$ system, a chemically accurate SRP functional ${ }^{10,11}$ has been created by taking a linear combination of 0.52 Perdew-Burke-Ernzerhof and 0.48 revised Perdew-Burke-Ernzerhof. To be able to compare the SCM to previous AIMD results, the underlying PES should be reproduced as closely as possible. This was done by performing ground state DFT calculations using the Vienna Ab initio Simulation Package (VASP). ${ }^{22-25}$ Calculations were performed using the Ultra-Soft (US) pseudopotentials ${ }^{23,25}$ provided by VASP, an $8 \times 8$ by $1 \Gamma$-centered k-point grid, an energy cutoff of $350 \mathrm{eV}$ for the planewave basis set, a Fermi-smearing with a temperature corresponding to $0.1 \mathrm{eV}$, and a convergence criterion of $0.1 \mathrm{meV}$.

Figure 1 shows a schematic overview of the $\mathrm{H}_{2}$ on the $\mathrm{Cu}(111)$ system. The $\mathrm{Cu}(111)$ slab has a FCC bulk structure with a cut in the $\langle 111\rangle$ direction and consists of four layers. These layers are defined using the lattice vectors $u$ and $v$ with $u$ being in the same direction as $x$, while $v$ has an angle of $\frac{\pi}{3}$ with $u$. The different layers are shifted in $u$ and $v$ of one third of both lattice vectors, and the interlayer distances are optimised for a $1 \times 1$ unit cell. A $3 \times 3$ unit cell was used in all calculations performed for computing coupling potentials.

\section{B. Static corrugation model}

The Static Corrugation Model (SCM $)^{13}$ is used to describe the effect of surface temperature due to surface atom displacements on the potential energy surface (PES) of a surface $(\vec{q})$ adsorbate $(\vec{r})$ system. This is realised by dividing the DFT PES $V_{D F T}(\vec{q}, \vec{r})$ into three terms: $V_{D F T}\left(\vec{q}^{i d}, \vec{r}\right)$ associated with an ideal surface, $V_{\text {strain }}$ associated with distorting a clean surface, and $V_{\text {coup }}$ associated with the change in the interaction of an adsorbate with the surface due to a surface atom displacement, as given by

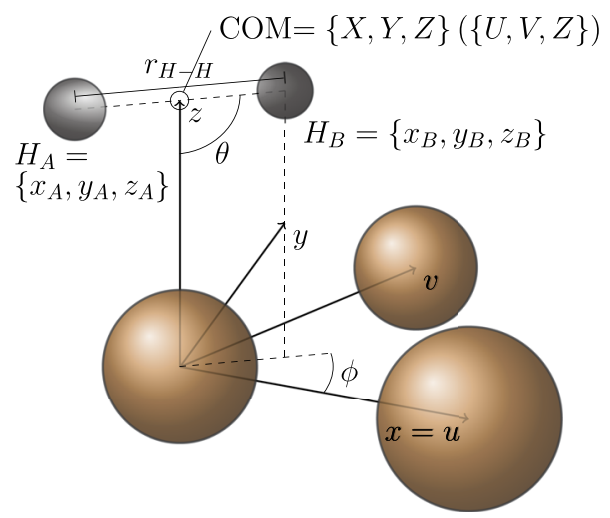

FIG. 1. Molecular coordinate system of $\mathrm{H}_{2}$ and lattice vectors for $\mathrm{Cu}(111)$ are shown. The first layer $\mathrm{Cu}$ atoms are indicated in brown, while the $\mathrm{H}$ atoms are indicated in gray. Indicated are the $z, y, x(=u)$, and $v$ unit vectors. $\mathrm{H}_{2}$ is described in both an atomic coordinate system, using the position of $H_{A}$ and $H_{B}$ on the $x, y$, and $z$ axis, and a molecular coordinate system using the position $X, Y, Z(U, V, Z)$ of the center of mass (COM) on the $x, y$, and $z(u, v$, and $z$ ) axis, respectively, together with bond distance $r_{\mathrm{H}-\mathrm{H}}$, polar angle $\theta$, and azimuthal angle $\phi$.

$$
\begin{aligned}
V_{D F T}(\vec{q}, \vec{r})= & V_{D F T}\left(\vec{q}^{i d}, \vec{r}\right)+V_{\text {coup }}\left(\vec{r}, \vec{q}^{i d} \rightarrow \vec{q}\right) \\
& +V_{\text {strain }}\left(\vec{q}^{i d} \rightarrow \vec{q}\right),
\end{aligned}
$$

where $\vec{q}$ describes the cartesian positions of all surface atoms, $\vec{q}^{i d}$ describes the ideal lattice positions in the same way, and $\vec{r}$ describes the cartesian positions of all adsorbate atoms (in this work only atoms $A$ and $B$ ). The strain potential $V_{\text {strain }}$ $\left(\vec{q}^{i d} \rightarrow \vec{q}\right)$ can be neglected for dynamics on a static surface because it is a constant if the surface configuration does not change during the dynamics. Using Eq. (1) and neglecting $V_{\text {strain }}$, the SCM thus approximates the full dimensional DFT PES according to

$$
\begin{aligned}
& V_{D F T}(\vec{q}, \vec{r}) \approx V_{S C M}\left(\vec{r}, \vec{q}^{i d} \rightarrow \vec{q}\right) \\
& \quad=V_{D F T}\left(\vec{q}^{i d}, \vec{r}\right)+V_{\text {coup }}\left(\vec{r}, \vec{q}^{i d} \rightarrow \vec{q}\right) .
\end{aligned}
$$

To be able to perform dynamics with the static corrugation potential, a continuous representation needs to be found for the ideal lattice $V_{D F T}\left(\vec{q}^{i d}, \vec{r}\right)$ as well as the coupling potential $V_{\text {coup }}\left(\vec{r}, \vec{q}^{i d} \rightarrow \vec{q}\right)$. It should be noted that HD-NNP could be a good alternative to obtain a continuous representation of the coupling potential $V_{\text {coup }}\left(\vec{r}, \vec{q}^{i d} \rightarrow \vec{q}\right)$. However, here we will continue along the lines of earlier work to be able to make a more thorough comparison. A representation of the SCM potential can be found by interpolating the DFT energies for the ideal surface using the Corrugation Reducing Procedure (CRP) method $^{14}$ and fitting the coupling potential with a functional form according to

$$
\begin{aligned}
V_{\text {coup }}\left(\vec{r}, \vec{q}^{\text {id }} \rightarrow \vec{q}\right) \\
\quad=\sum_{i}^{\vec{r}} \sum_{j}^{\vec{q}}\left(V_{\mathrm{H}-\mathrm{Cu}}\left(\left|\vec{r}_{i}-\vec{q}_{j}\right|\right)-V_{\mathrm{H}-\mathrm{Cu}}\left(\left|\vec{r}_{i}-\vec{q}_{j}^{i d}\right|\right)\right),
\end{aligned}
$$

where $\vec{r}_{i}$ is the cartesian position of adsorbate atom $i, \vec{q}_{j}$ is the cartesian position of surface atom $j$, and

$$
V_{\mathrm{H}-\mathrm{Cu}}(r)=V_{R y d}=(1-\rho(r)) V(r)+\rho(r) V\left(P_{7}\right),
$$

while

$$
V(r)=-e^{-P_{4}\left(r-P_{5}\right)} \cdot\left(\sum_{k=0}^{3} P_{k}\left(r-P_{5}\right)^{k}\right)
$$

and

$$
\rho(r)= \begin{cases}0 & \text { if } r<P_{6} \\ \frac{1}{2} \cos \left(\frac{\pi\left(r-P_{7}\right)}{P_{7}-P_{6}}\right)+\frac{1}{2} & \text { if } P_{6} \leq r \leq P_{7}, \\ 1 & \text { if } r>P_{7}\end{cases}
$$

where $P_{i}$ are the fitted parameters.

The SCM for $\mathrm{H}_{2}$ on $\mathrm{Cu}(111)$ from the previous work ${ }^{13}$ is extended by including an effective three-body interaction, a corrected surface stretching procedure, and a fitting procedure of the coupling potential including many more relevant surface and molecule configurations. We have recalculated the reaction probabilities for the model of Ref. 13 but using an improved implementation of the force scaling due to thermal expansion of the crystal via the stretching procedure. 
To include surface expansion due to surface temperature, the CRP potential of the system is stretched by contracting the $\mathrm{H}_{2} \mathrm{COM}$ vectors along the lattice vectors, instead of the Cartesian vectors of the atoms as was done previously. In this way, there are no additional small but unwanted contributions to the vibrational and rotational motion due to the stretching procedure. Hence, the full SCM potential becomes

$$
\begin{aligned}
V_{S C M}\left(\vec{r}, \vec{q}, \vec{q}^{i d}\right)= & V_{C R P}\left(\vec{r}^{i d}(\vec{r}), \vec{q}^{i d}\right) \\
& +\sum_{i}^{\vec{r}} \sum_{j}^{\vec{q}}\left(V_{\mathrm{H}-\mathrm{Cu}}\left(\left|\vec{r}_{i}-\vec{q}_{j}\right|\right)\right. \\
& \left.-V_{\mathrm{H}-\mathrm{Cu}}\left(\vec{r}_{i}^{i d}(\vec{r})-\vec{q}_{j}^{i d} \mid\right)\right),
\end{aligned}
$$

where $\vec{r}^{i d}(\vec{r})$ scales the expanded surface $\mathrm{H}_{2}$ coordinates $\vec{r}$ along the $\mathrm{COM} U$ and $\mathrm{V}$ coordinates to the ideal surface in such a way that they correspond to the same relative coordinates. The reaction probabilities using the original SCM model ${ }^{13}$ that are reported here using the improved implementation show no major differences compared to the previous results. The above methodology can be used for any 6D PES and is not limited to a CRP PES.

Here we define a $\mathrm{H}-\mathrm{Cu}$ interaction that is dependent not only on the distance between the $\mathrm{H}_{i}$ atom and $\mathrm{Cu}_{j}$ atom $\left(r_{i j}\right)$ but also on the $\mathrm{H}-\mathrm{H}$ bond distance $\left(r_{\mathrm{H}-\mathrm{H}}\right)$. This essentially describes the different behaviour of the $\mathrm{H}-\mathrm{Cu}$ interaction for $\mathrm{H}$ as a part of an $\mathrm{H}_{2}$ molecule (small $r_{\mathrm{H}-\mathrm{H}}$ ) and for $\mathrm{H}$ as an atom (large $r_{\mathrm{H}-\mathrm{H}}$ ). This was done by making all the parameters $P_{i}$ of the previous two-body SCM linearly dependent on $r_{\mathrm{H}-\mathrm{H}}$ as given by

$$
P_{i}= \begin{cases}P_{i, a} r_{\mathrm{H}-\mathrm{H}}^{\min }+P_{i, b} & \text { if } r_{\mathrm{H}-\mathrm{H}}<r_{\mathrm{H}-\mathrm{H}}^{\min } \\ P_{i, a} r_{\mathrm{H}-\mathrm{H}}+P_{i, b} & \text { if } r_{\mathrm{H}-\mathrm{H}}^{\min } \leq r_{\mathrm{H}-\mathrm{H}} \leq r_{\mathrm{H}-\mathrm{H}}^{\max }, \\ P_{i, a} r_{\mathrm{H}-\mathrm{H}}^{\max }+P_{i, b} & \text { if } r_{\mathrm{H}-\mathrm{H}}>r_{\mathrm{H}-\mathrm{H}}^{\max }\end{cases}
$$

where $r_{\mathrm{H}-\mathrm{H}}^{\min }$ and $r_{\mathrm{H}-\mathrm{H}}^{\max }$ are the cut-off values of the linear dependence set at the smallest and largest values of $r_{\mathrm{H}-\mathrm{H}}$ used in the fitting procedure. The resulting Rydberg function is then considered an $\mathrm{H}_{2}$-bond adapted Rydberg function.

\section{Quasi-classical dynamics of $\mathrm{H}_{2}$ and $\mathrm{D}_{2}$ on $\mathrm{Cu}(111)$}

Using either the CRP $\left(V_{C R P}\right)$ or SCM PES $\left(V_{S C M}\right)$, it is possible to describe the dissociation of $\mathrm{H}_{2}$ or $\mathrm{D}_{2}$ on an ideal or non-ideal (with displaced $\mathrm{Cu}$ atoms) $\mathrm{Cu}(111)$ surface. The PES itself is not a directly measurable observable, so instead we performed molecular beam simulations. These simulations were performed using the Quasi-Classical (QC) dynamics ${ }^{11,15}$ method. Initial conditions are determined by using a MonteCarlo sampling scheme, where zero point energies for the $\mathrm{H}_{2}$ bond were taken into account only for the initial configurations. Then the system is propagated classically and the resulting trajectory is finally analyzed. We performed 20000 trajectories for each choice of incidence energy $E_{i}$, vibrational state $v$, and rotational state $J$.

The initial conditions for the molecule are generated by first calculating the rovibrational energy levels of the $\mathrm{H}_{2}$ or $\mathrm{D}_{2}$ molecule for the used PES using the Fourier grid Hamiltonian method. ${ }^{26}$ To get the QC distribution for the $\mathrm{H}-\mathrm{H}$ bond distance $r_{\mathrm{H}-\mathrm{H}}$ of the $\mathrm{H}_{2}$ molecule, the gasphase $\mathrm{H}_{2}$ molecule was propagated, and positions and momenta were recorded, for one complete phase in its vibration using a constant time step. The initial atomic positions and velocities were then chosen using standard Monte-Carlo methods. The $\phi$ and $\theta$ angles are chosen from a uniform random distribution in the range $[0,2 \pi]$ and $[0, \pi]$, respectively. Angular velocities are chosen according to the quantized angular momentum $L^{2}=J(J+1) \hbar^{2}$ with the angle $\theta_{L}$ between the angular momentum vector and the surface normal chosen randomly but constrained by $\theta_{L}=\pi$ if $\mathrm{J}=0$ and $\cos \left(\theta_{L}\right)=\frac{m_{J}}{\sqrt{J(J+1)}}$ if $\mathrm{J} \geq 1$. The $\mathrm{Z}$ component of the COM velocity is set to correspond to a kinetic energy of $E_{i}$. The initial COM position is then shifted $9 \AA$ in $Z$ away from the surface $(Z=9 \AA)$ while the $C O M$ position along the $F C C(111)$ surface is given by $X=\tilde{U}+\frac{1}{2} \tilde{V}$ and $Y=\frac{1}{2} \sqrt{3} \tilde{V}$ where $\tilde{U}$ and $\tilde{V}$ are chosen from a uniform random distribution in the range $[0$, $a$ ] with $a$ being the lattice constant. This process was identical to earlier work. ${ }^{9,11,13,15,16}$

The SCM uses the surface atom positions of both the ideal lattice and the corrugated lattice. The ideal lattice is constructed in the same way as the DFT slab used for constructing the CRP with the exception that no periodic boundary conditions are used. Instead, for each trajectory, the initial COM position is projected onto the surface plane and only copper atoms that are from the first three layers and within a radius of $12 \AA$ around the projected COM are considered. The corrugated lattice is constructed from this ideal lattice in three steps. First, the surface expansion along the $\mathrm{u}$ and $\mathrm{v}$ lattice vectors is introduced. The relative experimentally ${ }^{27}$ observed expansion is applied to the lattice constant from the CRP potential. Second, the interlayer spacings are adjusted in a similar fashion: the experimentally observed relative expansion or contraction ${ }^{28}$ in the interlayer spacings is applied to the interlayer spacings used in the DFT slab of the respective CRP potential. Finally, for each surface atom, a random direction is chosen and the magnitude of the displacement is randomly selected from an appropriate surface temperature dependent gaussian distribution based on Debye-Waller factors. ${ }^{29}$

Once the initial conditions are defined, only the molecule is propagated according to Hamilton's equations of motion with the following hamiltonian (in atomic units):

$$
H=\frac{p_{A}^{2}(t)}{2 m_{A}}+\frac{p_{B}^{2}(t)}{2 m_{B}}+V(R(t)),
$$

where $p_{A}(t)$ and $p_{B}(t)$ are the momenta of atoms $\mathrm{A}$ and $\mathrm{B}$, respectively, at time $t$ and $V(R(t))$ is the potential energy at the cartesian position $R(t)$ of both atoms $A$ and $B$. The propagation is performed using the predictor-corrector method of Bulirsch and Stoer. ${ }^{30}$ A trajectory is considered to be reactive when the $\mathrm{H}$ atoms are separated by more than $2.25 \AA$ before the time cutoff of $20 \mathrm{ps}$ and considered non-reactive otherwise. When a trajectory is non-reactive, the molecule is either trapped as a molecule on the surface or has scattered to the same or a different rovibrational state which is called elastic or inelastic scattering, respectively. No significant contribution of trapped molecules was found during this study and are therefore not reported. The final rovibrational state is determined by binning first to the rotational state based on angular 
momentum and then to the closest vibrational state in energy with the previously determined rotational state. The results have been obtained for the $\mathrm{H}_{2}$ and $\mathrm{D}_{2}$ on the $\mathrm{Cu}(111)$ system with both an ideal lattice, and a displaced and expanded lattice at a finite surface temperature of $\mathrm{T}_{\mathrm{s}}=925 \mathrm{~K}$.

\section{RESULTS AND DISCUSSION}

\section{A. Coupling potential}

To be able to verify and improve the fit of the coupling potentials used in the previous work, ${ }^{13}$ we repeated the computation of the coupling potentials, but this time with the same DFT setup as was used for AIMD calculations. ${ }^{9}$ A comparison with these AIMD results and the SCM results can show the importance of surface motion compared to surface displacement. To improve the old SCM model, we also fitted the new SCM model to the coupling potential for 15113 configurations instead of 153.

To test the previous assumption that the coupling potential can be approximated with only two-body interactions, i.e., $\mathrm{H}-\mathrm{Cu}$ interactions, we computed the coupling potential at configurations $\vec{q}_{i d} \rightarrow \vec{q}_{i d}+\vec{Q}_{1}+\vec{Q}_{2}$ where two displacements $\vec{Q}_{1}$ and $\vec{Q}_{2}$ were made to isolate $\mathrm{H}-\mathrm{Cu}-\mathrm{Cu}$ effective three-body interactions. To compute the $\mathrm{H}-\mathrm{H}-\mathrm{Cu}$ effective three-body interactions, the configurations with the displacements $\vec{q}_{i d} \rightarrow \vec{q}_{i d}+\vec{Q}$ and $\vec{r} \rightarrow \vec{r}^{\prime}$ were used. This has two advantages, namely, fitting these coupling potentials will result in an effective three-body interaction but at the same time it allows us to test how well the two-body approximation performs.

Coupling potentials presented here are computed exactly from DFT calculations according to

$$
\begin{aligned}
& V_{\text {coup }}\left(\vec{r}, \vec{q}_{i d} \rightarrow \vec{q}_{i d}+\vec{Q}\right)=V_{D F T}\left(\vec{r}, \vec{q}_{i d}+\vec{Q}\right) \\
& -V_{D F T}\left(\vec{r}, \vec{q}_{i d}\right)-V_{\text {strain }}\left(\vec{q}_{i d} \rightarrow \vec{q}_{i d}+\vec{Q}\right),
\end{aligned}
$$

where

$$
\begin{aligned}
V_{\text {strain }}\left(\vec{q}_{i d} \rightarrow \vec{q}_{i d}+\vec{Q}\right)= & V_{D F T}\left(\vec{r}_{\text {gas }}, \vec{q}_{i d}+\vec{Q}\right) \\
& -V_{D F T}\left(\vec{r}_{\text {gas }}, \vec{q}_{i d}\right),
\end{aligned}
$$

with $\vec{r}_{\text {gas }}$ indicating that the $\mathrm{H}_{2}$ molecule has been moved $6 \AA$ away from the surface such that there is essentially no interaction between $\mathrm{H}_{2}$ and the surface. Here the displacement $\vec{r} \rightarrow \vec{r}^{\prime}$ is understood as simply evaluating the coupling potential at $\vec{r}^{\prime}$ instead of $\vec{r}$.

We first considered the $\mathrm{H}-\mathrm{Cu}-\mathrm{Cu}$ three-body coupling potential. For these coupling potentials, the $\mathrm{H}_{2}$ was placed at the barrier position of the bridge, top, or HCP site. Two atoms were selected from the first two layers in the surface considering all permutations and symmetries. These were then either moved in all possible combinations of the directions $x(=u), y, v$, and $z$, as well as both atoms moving towards and away from the $\mathrm{H}_{2} \mathrm{COM}$. The displacements have a magnitude from $-0.3 \AA$ until $0.3 \AA$ with a step of $0.1 \AA$. There are too many permutations to discuss all of them, but a representative selection is discussed next.

Figure 2 shows the coupling potentials of the surface displacements of the two surface atoms closest to the $\mathrm{H}_{2} \mathrm{COM}$ perpendicular to the surface and $\mathrm{H}_{2}$ at the lowest barrier for this system (bridge site barrier). The displacements $\vec{Q}_{1}$ and $\vec{Q}_{2}$ are in this case symmetric due to the mirror plane along the $\mathrm{H}$ bond, meaning that the values for the displacements can be swapped without changing the coupling potential. What is interesting to note here is that the lowest coupling potential is

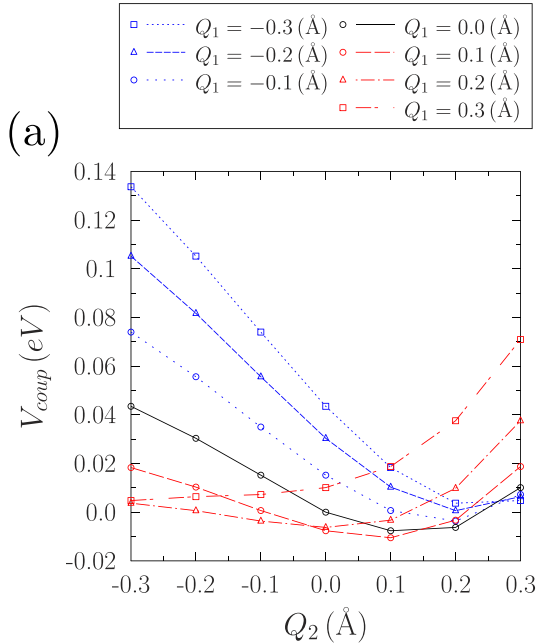

(c)

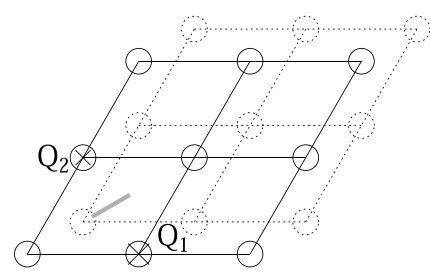

(b)

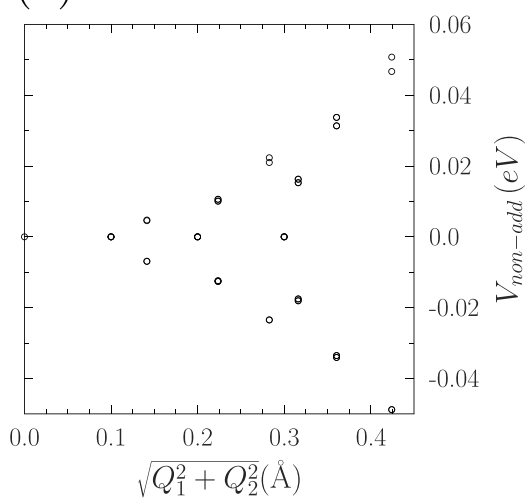

(d)

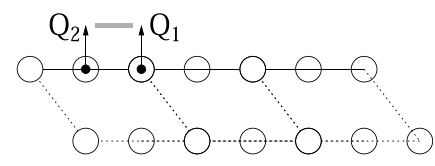

FIG. 2. (a) shows $V_{\text {coup }}$ for displacements along $\vec{Q}_{1}$ and $\vec{Q}_{2}$ where black, red, and blue show no, a positive, and a negative displacement along $\vec{Q}_{1}$, respectively. The displacements $\vec{Q}_{1}$ and $\vec{Q}_{2}$ are defined in (c) and (d) by showing a top and front view of the system, respectively, where $\mathrm{Cu}$ atoms are indicated using circles and $\mathrm{H}_{2}$ by the gray bar. (b) shows the non-additivity [see Eq. (13)] of the coupling potentials presented in (a). $Q_{1}$ and $Q_{2}$ in (a) and (b) refer to the magnitudes $\left|\vec{Q}_{1}\right|$ and $\left|\vec{Q}_{2}\right|$, respectively, while $Q_{1}$ and $Q_{2}$ in (c) and (d) are the vectors $\vec{Q}_{1}$ and $\vec{Q}_{2}$. 
not at the ideal lattice positions (with the displacements at $0 \AA$ ) but with the surface atoms slightly moved out of the surface, indicating a puckering ${ }^{31}$ effect. This puckering effect cannot be taken into account using the SCM (where static surface configurations are Monte-Carlo sampled randomly); however, it is not expected to have a large influence on dynamics due to the large mass difference between $\mathrm{H}_{2}$ and $\mathrm{D}_{2}$ with $\mathrm{Cu}$ as well as the high velocities and consequent short interaction time of $\mathrm{H}_{2}$.

The configuration with the most favorable coupling potential in Fig. 2, at $-10.5 \mathrm{meV}$, is when both atoms are displaced by $+0.1 \AA$ along $\vec{Q}_{1}$ and $\vec{Q}_{2}$. When only one atom is displaced, while the other is at its ideal lattice position, the coupling potential is $7.5 \mathrm{meV}$. From a perspective where we only consider additive interactions [see Eq. (7)], this cannot be explained as both surface atoms should then interact independently. This means that a three (or more)-body interaction is present, or in other words there is non-additivity of the coupling potential due to the surface displacements of two or more $\mathrm{Cu}$ atoms. The non-additivity of the coupling potential [see Eq. (10)] is defined as the difference between the coupling potential of displacements $\vec{Q}_{1}$ and $\vec{Q}_{2}$ with the sum of the coupling potentials of only $\vec{Q}_{1}$ and only $\vec{Q}_{2}$, as given by

$$
\begin{aligned}
V_{\text {nonadd }}\left(\vec{r}, \vec{q}^{i d} \rightarrow \vec{q}^{i d}+\vec{Q}_{1}+\vec{Q}_{2}\right) \\
=V_{\text {coup }}\left(\vec{r}, \vec{q}^{i d} \rightarrow \vec{q}^{i d}+\vec{Q}_{1}+\vec{Q}_{2}\right) \\
\quad-V_{\text {coup }}\left(\vec{r}, \vec{q}^{i d} \rightarrow \vec{q}^{i d}+\vec{Q}_{1}\right) \\
-V_{\text {coup }}\left(\vec{r}, \vec{q}^{i d} \rightarrow \vec{q}^{i d}+\vec{Q}_{2}\right) .
\end{aligned}
$$

While this non-additivity can never be modeled with an additive interaction, there are good arguments to believe that they are not very important. First of all, the additive interaction can be fitted including the configurations with non-additivity, resulting in an effective non-additive interaction that takes into account the average non-additivity. The distribution of the non-additivity was found to have a mean of $-0.5 \mathrm{meV}$ and a standard deviation (SD) of $6.3 \mathrm{meV}$ which means that on average the correct coupling potential should be reproduced only introducing a slightly bigger spread in the modeled coupling potentials. Furthermore, the configurations where there is a lot of non-additivity are when there are two specific surface atoms that both have a large displacement. While it is certainly true that a single displacement of such a magnitude is not extremely unlikely, having two such large displacements is even less common and therefore decreases the probability of sampling this error. Finally, in a realistic configuration of a non-ideal surface, all surface atoms are displaced, and combining this with the fact that the non-additivity introduces an error that is on average zero, there is an even smaller mean error under these realistic conditions. Even without these arguments, under these circumstances, the absolute mean error of this non-additivity is well within the accuracy one can expect for the underlying DFT.

In Fig. 3, a summary is presented of the coupling potentials and their respective non-additivity. The green lines are added as a reference to show the approximate accuracy of the

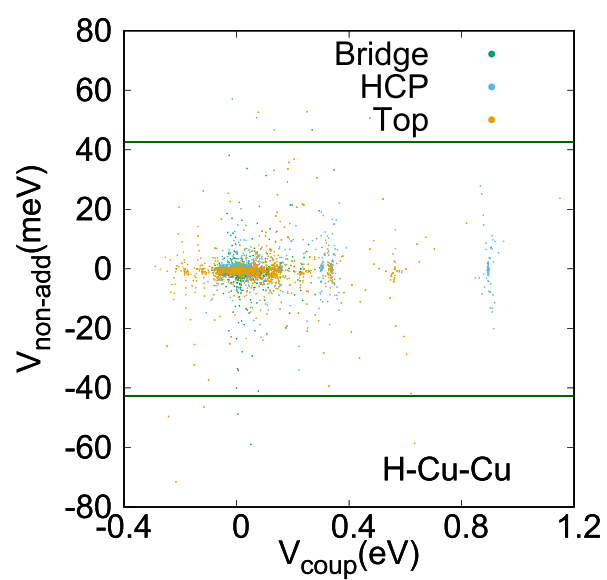

FIG. 3. The non-additivity of $V_{\text {coup }}$ is correlated with the value of $V_{\text {coup }}$ for configurations with two surface atom displacements $(\mathrm{H}-\mathrm{Cu}-\mathrm{Cu}$ three-body interactions) and $\mathrm{H}_{2}$ at the bridge (green), $\mathrm{HCP}$ (blue), and top (orange) site. The potential at which the nonadditivity exceeds chemical accuracy is shown as a reference in dark green [these $43 \mathrm{meV}$ lines correspond to more than 6 times the SD we found in the DFT data $(6.3 \mathrm{meV})]$.

underlying DFT method used. The behavior of the $\mathrm{H}-\mathrm{Cu}-\mathrm{Cu}$ non-additivity is essentially independent of the chosen site (bridge, $\mathrm{HCP}$, and top) for $\mathrm{H}_{2}$. Almost all coupling potentials of the $\mathrm{H}-\mathrm{Cu}-\mathrm{Cu}$ type have a non-additivity smaller than the accuracy of the DFT method, which means that any subsequent improvement of the fitting quality is not guaranteed to yield a more realistic coupling potential.

The $\mathrm{H}-\mathrm{H}-\mathrm{Cu}$ coupling potentials describe how the coupling potential changes when the $\mathrm{H}_{2}$ bond distance changed $\left(\vec{r} \rightarrow \vec{r}^{\prime}\right)$ whilst also displacing a single $\mathrm{Cu}$ atom $(\vec{Q})$ at the same time. These coupling potentials were selected in a similar fashion as the $\mathrm{H}-\mathrm{Cu}-\mathrm{Cu}$ coupling potentials. Instead of selecting two surface atoms, only one was selected and the same displacements were used for the single atom in combinations with increasing and decreasing the $\mathrm{H}_{2}$ bond distance from $-0.3 \AA$ until $0.3 \AA$ with respect to the barrier position in steps of $0.1 \AA$. Other degrees of freedom of $\mathrm{H}_{2}$ were sampled in the same way. Consequently, the $\mathrm{H}-\mathrm{H}-\mathrm{Cu}$ non-additivity is given by

$$
\begin{aligned}
V_{\text {nonadd }} & \left(\vec{r} \rightarrow \vec{r}^{\prime}, \vec{q}^{i d} \rightarrow \vec{q}^{i d}+\vec{Q}\right) \\
& =V_{\text {coup }}\left(\vec{r}^{\prime}, \vec{q}^{i d} \rightarrow \vec{q}^{i d}+\vec{Q}\right) \\
& -V_{\text {coup }}\left(\vec{r}, \vec{q}^{i d} \rightarrow \vec{q}^{i d}+\vec{Q}\right) \\
& -V_{\text {coup }}\left(\vec{r}^{\prime}, \vec{q}^{i d} \rightarrow \vec{q}^{i d}\right),
\end{aligned}
$$

where $V_{\text {coup }}\left(\vec{r}^{\prime}, \vec{q}^{i d} \rightarrow \vec{q}^{i d}\right)=0$ due to the lack of surface displacements. The $\mathrm{H}-\mathrm{H}$ distance $r_{\mathrm{H}-\mathrm{H}}$ ranges from $\mathrm{H}_{2}$ having dissociated at very large bond distances to the bond distance being so short that almost all available energy in the system has gone into this compression. Unlike the $\mathrm{H}-\mathrm{Cu}-\mathrm{Cu}$ interactions, the $\mathrm{H}-\mathrm{H}-\mathrm{Cu}$ interactions cannot be reproduced within chemical accuracy using a two-body $\mathrm{H}-\mathrm{Cu}$ interaction. Figure 4 shows the non-additivity of the $\mathrm{H}-\mathrm{H}-\mathrm{Cu}$ coupling potentials, and there is a much broader distribution 


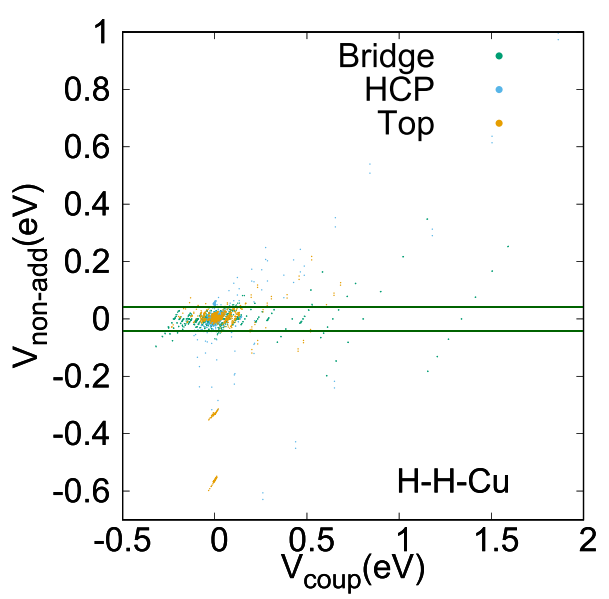

FIG. 4. The same as in Fig. 3 but for configurations with one surface atom displacement combined with the $\mathrm{H}-\mathrm{H}$ bond not at the equilibrium distance ( $\mathrm{H}-\mathrm{H}-\mathrm{Cu}$ three-body interactions).

with a mean of $-5.0 \mathrm{meV}$ and a SD of $74.6 \mathrm{meV}$, with some non-additivities being more than ten times chemical accuracy. Generally, we find that the non-additivity and coupling potential are linearly dependent on the $\mathrm{H}_{2}$-bond distance. Such linear dependence has been seen before for the vibrational coupling of diatomics interacting with atoms and other diatomics. ${ }^{32,33}$ In contrast to the $\mathrm{H}-\mathrm{Cu}-\mathrm{Cu}$ non-additivity, there is a slight difference of the $\mathrm{H}-\mathrm{H}-\mathrm{Cu}$ non-addivity for $\mathrm{H}_{2}$ at different sites. There is a larger spread of the nonadditivity for $\mathrm{H}_{2}$ at the bridge site, while for some configurations at the top site, there is a very large non-additivity, eventhough there is a small coupling potential. Due to the large non-additivity for $\mathrm{H}-\mathrm{H}-\mathrm{Cu}$ interactions, we conclude that it is necessary to make use of the $\mathrm{H}_{2}$-bond adapted Rydberg function.

\section{B. Parameters for the static corrugation model}

The coupling potential discussed above was fitted using the $\mathrm{H}_{2}$-bond adapted Rydberg function using the fitting procedure described above.

All fits take the $\mathrm{H}-\mathrm{Cu}-\mathrm{Cu}$ interactions into account only effectively, while the $\mathrm{H}-\mathrm{H}-\mathrm{Cu}$ interactions are taken into account through the $r_{\mathrm{H}-\mathrm{H}}$ dependence of the parameters $P$ [see Eq. (8)]. The fitting procedure was repeated with four different sets of constraints. First, the most relaxed case where $\mathrm{H}-\mathrm{H}-\mathrm{Cu}$ three-body interactions are taken into account and two different sets of parameters were assigned, one for the first and the other for the second surface layer copper atoms involved in the $\mathrm{H}-\mathrm{Cu}$ interaction (3-Body 2-Layer). Then there is the case where a single set of parameters is used for both layers (3-Body 1-Layer) and the case where all non-additive interactions are taken into account only effectively (2-Body 2-Layer). Finally, the fit was also performed constraining both the parameters to a single set and taking non-additive interactions into account only effectively (2-Body 1-Layer). This is thus essentially a refit of the old $\mathrm{SCM}^{13}$ using the new and vastly extended set of DFT dataset and consequent coupling potential. The best fit is for the 3-Body 2-Layer case with a close second place for the 3-Body 1-Layer case. The other two cases have a very similar root-mean-square error (RMSE) as reported in the literature
TABLE I. An overview of the different fitting constraints and the corresponding names.

\begin{tabular}{lccc}
\hline \hline Fit & $\begin{array}{c}\text { 3-Body } \\
\text { interactions }\end{array}$ & $\begin{array}{c}\text { Different } \\
\text { parameter sets }\end{array}$ & $\begin{array}{c}\text { RMSE } \\
(\mathrm{meV})\end{array}$ \\
\hline 3-Body 2-Layer & Yes & Yes & 29.4 \\
2-Body 2-Layer & No & Yes & 62.4 \\
3-Body 1-Layer & Yes & No & 42.7 \\
2-Body 1-Layer & No & No & 66.6 \\
\hline \hline
\end{tabular}

for other methods such as the ReaxFF. ${ }^{34}$ In the case of the $\mathrm{H}_{2}$-bond adapted Rydberg function, the plots are for several different $\mathrm{H}_{2}$-bond distances as shown in Table III. An overview of the RMSE is presented in Table I, and the parameters for all 1-Layer cases are given in Table II.

Comparing the $V_{\mathrm{H}-\mathrm{Cu}}$ interaction from the previous work ${ }^{13}$ with the 2-Body 1-Layer fit to the new DFT dataset in Fig. 5, the interaction is weaker than before but qualitatively very similar. The position of the maximum is shifted to about half a Bohr shorter $\mathrm{H}-\mathrm{Cu}$ distance, while the position of the well is still the same. When instead the fit is performed with different parameters for different layers, the first layer interaction is shifted to a lower energy but the barrier and equilibrium position are still very similar in position and height. The second layer interaction, on the other hand, becomes much more repulsive at a low distance and slightly more attractive at high distances. It should be noted here that the repulsive wall is not a regime that is sampled as it is not possible for an $\mathrm{H}$ atom to get this close to a second layer atom. The three-body interaction is represented in Fig. 5 by showing the energy dependence of the $\mathrm{H}-\mathrm{Cu}$ distance at several fixed values of the $\mathrm{H}-\mathrm{H}$ distance as discussed in Table III (green curves). The general shape of the interaction is the same at every $\mathrm{H}-\mathrm{H}$ distance, but at high $\mathrm{H}-\mathrm{H}$ distances, the barrier is much higher while the well is much lower in energy. There is also a slight broadening effect such that the well is shifted to smaller $\mathrm{H}-\mathrm{Cu}$ distances and the barrier to larger $\mathrm{H}-\mathrm{Cu}$ distances. At very small $\mathrm{H}-\mathrm{H}$ distances, the $V_{\mathrm{H}-\mathrm{Cu}}$ interaction becomes almost completely repulsive. Surprisingly, the bridge site, which has the lowest $\mathrm{H}-\mathrm{H}$ distance and therefore the most repulsive $\mathrm{H}-\mathrm{Cu}$ interaction, actually has the lowest barrier for reaction. These two statements are not contradictory because the $\mathrm{H}-\mathrm{Cu}$ interaction

TABLE II. Fitted SCM parameters where units of length are in Bohr and units of energy are in Hartree. Columns $a$ and $b$ for the 3-Body interactions refer to $P_{i, a}$ and $P_{i, b}$ from Eq. (8), respectively.

\begin{tabular}{lcrrr}
\hline \hline & & & \multicolumn{2}{c}{ 3-Body } \\
\cline { 3 - 5 } & Reference 13 & 2-Body & \multicolumn{1}{c}{$b$} & \multicolumn{1}{c}{$a$} \\
\hline$P_{0}$ & -0.0303 & -0.0339 & -0.0704 & 0.0166 \\
$P_{1}$ & 0.1035 & 0.1024 & 0.0235 & 0.0287 \\
$P_{2}$ & -0.0692 & -0.0802 & -0.0633 & -0.0072 \\
$P_{3}$ & $\ldots$ & 0.0111 & 0.0272 & -0.0064 \\
$P_{4}$ & 1.2744 & 1.2929 & 1.2910 & -0.0031 \\
$P_{5}$ & 2.3005 & 2.3023 & 2.2897 & 0.0236 \\
$P_{6}$ & 7.4442 & 7.4400 & 7.4402 & 0.0008 \\
$P_{7}$ & 7.4636 & 10.4600 & 10.4601 & 0.0000 \\
\hline \hline
\end{tabular}




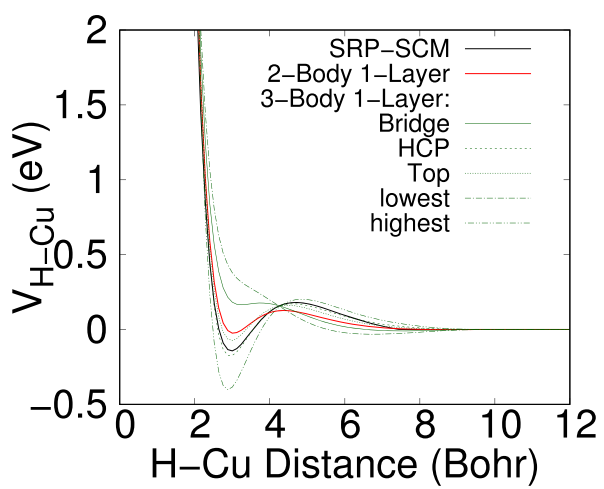

FIG. 5. The $V_{\mathrm{H}-\mathrm{Cu}}$ interaction is shown as a function of the $\mathrm{H}-\mathrm{Cu}$ distance $r_{\mathrm{H}-\mathrm{Cu}}$ for the SCM from Ref. 13 (black), the 2-Body 1-Layer fit (red), and the 3-Body 1-Layer fit (green). The three-body interaction is represented by showing the potential dependence on the $\mathrm{H}-\mathrm{Cu}$ distance at specific $\mathrm{H}-\mathrm{H}$ distances according to Table III.

only includes the influence of the $\mathrm{H}-\mathrm{H}$ distance on $V_{\text {coup }}$ and not the $\mathrm{H}-\mathrm{H}$ interaction itself, which is included in the CRP potential $\left(V_{C R P}\right)$. The described features suggest that the fitted potential is at least qualitatively in agreement with the properties of the PES and the RMSE suggests that there is also a quantitative agreement.

While the RMSE of the 3-Body 2-Layer fit suggests it is the best fit, the shape of the $V_{\mathrm{H}-\mathrm{Cu}}$ interaction potential tells a different story. The problem in this case is the fit coverage: there are almost no first layer interactions in the 8 to 10 Bohr region so there is no constraint on the fitting procedure to keep the $V_{\mathrm{H}-\mathrm{Cu}}$ interaction at a sensible value. Dynamical calculations using the underfitted 3-Body 2-Layer $V_{\mathrm{H}-\mathrm{Cu}}$ yielded non-sensible results and are not presented in this work.

In the rest of this work, we only consider a single set of parameters used for both the first and second layer $\mathrm{H}-\mathrm{Cu}$ interaction instead. Using that fitting procedure to fit the new set of coupling potentials, we have successfully fitted an $\mathrm{H}_{2}$ bond adapted Rydberg function. The resulting RMSE of the 3-Body 1-Layer fit is significantly improved compared to the RMSE of the SCM from previous work when applied to the new DFT dataset and is within the same accuracy as the DFT data used for the fitting.

\section{Dynamics on different potential energy surfaces}

To properly compare the newly developed SCM with the previous SCM, ${ }^{13}$ which uses two comparable but slightly different DFT functionals, we first compared the results using the 6D CRP Born-Oppenheimer Static Surface (BOSS) interpolations for the two used functionals. The previous SCM used the SRP functional by Díaz et al. ${ }^{15}$ for which

TABLE III. An overview of the different $\mathrm{H}-\mathrm{H}$ distances used in this section.

\begin{tabular}{lc}
\hline \hline Name & H-H distance $(\AA)$ \\
\hline Bridge & 1.025 \\
HCP & 1.547 \\
Top & 1.402 \\
Lowest $\left(r_{\text {min }}\right)$ & 0.725 \\
Highest $\left(r_{\text {max }}\right)$ & 1.847 \\
\hline \hline
\end{tabular}

we refer to the CRP interpolation with SRP-BOSS (in red), and the newly developed SCM uses the SRP48 functional by Nattino et $\mathrm{al}^{35}$ for which a CRP interpolation SRP48BOSS (in black) was made by Mondal et al. ${ }^{16}$ Using these two different PESs, dynamical scattering simulations were performed for $\mathrm{D}_{2}$ on $\mathrm{Cu}(111)$. While reaction probabilities have been reported before, the rovibrationally (in)elastic scattering probabilities have not. Only the initial rovibrational states $(\mathrm{v}=0, \mathrm{~J}=0),(\mathrm{v}=0, \mathrm{~J}=11)$, and $(\mathrm{v}=1, \mathrm{~J}=6)$ are discussed here specifically, and a general overview is given for the other states computed (for which the results are available digitally).

The general trend for $\mathrm{QC}$ reaction probabilities of $\mathrm{D}_{2}$ on $\mathrm{Cu}(111)$ is that at low incidence energies, there is no reaction and as the incidence energy increases the reaction probability increases until it reaches a maximum value called the saturation value. As described previously, ${ }^{9}$ the general curve of the reaction probability can be described with a modified logistics function. When there is no reaction, there can either be elastic scattering or inelastic scattering. At low incidence energies, the elastic scattering dominates as there is not yet enough energy or coupling available to cause a rovibrational excitation, while at high energies, the inelastic scattering dominates as there is more coupling due to the corrugation and anisotropies close to the surface. It is also possible for the $\mathrm{H}_{2}$ molecule to change its momentum along the surface lattice vectors when scattering, which is called diffraction. The effect of diffraction was not considered in the sense that the reported observables are summed over the final diffraction states.

The differences between the reaction and (in)elastic scattering probabilities predicted by the two PESs (see the black and red curves in Fig. 6) are dependent on the rovibrational state. For the $(v=0, J=0)$ state, the reaction and (in)elastic scattering probabilities are different below $0.9 \mathrm{eV}$, while for

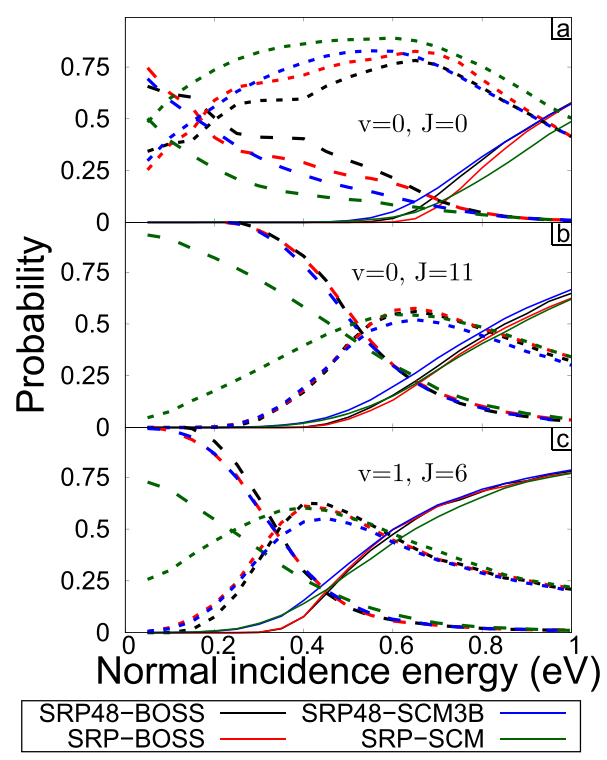

FIG. 6. The state-specific reaction (solid curves), elastic scattering (long dashed curves), and inelastic scattering (short dashed curves) probabilities are shown as a function of the incidence energy for SRP48-BOSS (black), SRP48SCM3B (blue), SRP-BOSS (red), and SRP-SCM (green). (a)-(c) show the $(\mathrm{v}=0, \mathrm{~J}=0),(\mathrm{v}=0, \mathrm{~J}=11)$, and $(\mathrm{v}=1, \mathrm{~J}=6)$ rovibrational state-specific reaction and (in)elastic scattering probabilities, respectively. 
the $(v=0, J=0)$ state, the reaction and (in)elastic scattering probabilities are different above $0.5 \mathrm{eV}$, and for the $(\mathrm{v}=1$, $J=6$ ) state, the reaction probabilities are the same for all energies whereas the (in)elastic scattering probabilities deviate below $0.5 \mathrm{eV}$.

The reaction and rovibrationally (in)elastic scattering probabilities for the $(\mathrm{v}=0, \mathrm{~J}=0)$ initial state are shown in Fig. 6(a) and we will now discuss $(\mathrm{v}-0, \mathrm{~J}=0)$ in detail first. For incidence energies above $0.9 \mathrm{eV}$, the probabilities are almost the same for the two PESs. The probabilities for elastic scattering are the same above $0.7 \mathrm{eV}$, while the reaction probability is lower in the case of the SRP-BOSS PES and the inelastic scattering probabilities are higher. This means that in the region of $0.7 \mathrm{eV}-0.9 \mathrm{eV}$, there is a different preference to either react or scatter for the SRP48-BOSS PES compared to the SRP-BOSS PES. In the case of the SRP48-BOSS PES, the preference is more towards the reaction, while in the case of the SRP-BOSS PES, the incidence energy is converted into some rovibrational excitations and the preference is towards rovibrationally inelastic scattering. At even lower incidence energies, the rovibrationally inelastic scattering probability for the SRPBOSS PES is lower only for the lowest incidence energy, and for all other incidence energies, it is higher than the rovibrationally inelastic scattering probability of the SRP48-BOSS PES.

For the rotationally excited state $(\mathrm{v}=0, \mathrm{~J}=11)$, as shown in Fig. 6(b), there is almost no difference between the two PESs except for a small broadening of the reaction probability in the case of the SRP-BOSS PES compared to the SRP48BOSS PES. The lower reaction probability is mostly compensated by a higher inelastic scattering for the SRP-BOSS PES.

On the other hand, the two PESs yield very similar reaction probabilities for the vibrationally excited state $(\mathrm{v}=1$, $\mathrm{J}=6$ ), while the SRP-BOSS PES inelastic and elastic scattering probability curves cross earlier compared to the SRP48-BOSS PES as shown in Fig. 6(c). The general trend of all computed rovibrational states is that as more vibrational energy is added, the reaction probabilities become almost identical between the SRP48-BOSS and SRP-BOSS PES while adding more rotational energy causes the elastic and inelastic scattering probabilities to be more comparable.

Here we argue that these two effects are distinct features of the PESs based on a normal mode analysis performed along the minimum energy path (MEP) for both PESs as given in the Appendix. The difference between the widths of the reaction probabilities for the SRP and SRP48 are mostly determined by how much vibrational energy (in r) is added initially in $\mathrm{H}_{2}$. If vibrational energy is added, the details of the intrinsic curvature (or how the forces along $\mathrm{Z}$ and $\mathrm{r}$ change) of the PES along the MEP towards the barrier become less important because there is more energy available. The (extrinsic) curvature describing the geometric shape of the reaction path is essentially identical for the two PESs. We note that both functionals yield very similar barrier heights and locations for this system as well as near identical MEPs. Therefore, we argue that it is the intrinsic curvature, constrained in $r$ and $\mathrm{Z}$, that determines the different reaction probability widths for the SRP-BOSS and SRP48-BOSS. Previously, it has been reported $^{36}$ that the energetic corrugation can also have an influence on the reaction probability width, but that would not directly explain the strong dependence on the initial vibrational state. If instead rotational energy is added, the intrinsic curvature, in $r$ and $\mathrm{Z}$, of the PES towards the barrier is still important. A similar argument can be made for the energy at which the rovibrationally elastic and inelastic scattering probability curves cross, where it is mostly the anisotropy in $\theta$ and $\phi$ that determines if the rovibrational state changes. If the molecule is rotating relatively fast, it feels an "average" of the potential in $\theta$ and therefore the exact shape of PES becomes less important.

Both PESs were designed to reproduce molecular beam experiments, where the effective barrier height is the most important property of the PES, but if there is not enough energy available to sample the effective barrier, the shape of the PES towards the barrier is also important.

\section{Comparison of different static corrugation models}

In this section, a comparison is made between the new SCM based on the PES from Mondal et al. ${ }^{16}$ (SRP48SCM3B) and a SCM from the previous work ${ }^{13}$ based on the PES from Diaz et al. ${ }^{15}$ (SRP-SCM). Both SCMs were computed for a surface temperature of $925 \mathrm{~K}$ including both surface displacements and surface expansion as described previously.

Figure 6(a) shows the effect of using the SCM compared to the BOSS model for the rovibrational ground state $(\mathrm{v}=0, \mathrm{~J}$ $=0$ ). For the SRP-SCM PES, there is a large broadening ${ }^{8,13,37}$ of the reaction probability at both low and high reaction probabilities, while the SRP48-SCM3B PES only shows increased reaction probabilities at low incidence energies. The reaction probability of the SRP48-SCM3B PES starts to increase earlier compared to SRP-SCM, but after $0.8 \mathrm{eV}$ of incidence energy, the slope is essentially the same as for the SRP-SCM PES. The rovibrationally elastic and inelastic scattering probability curves for the SCM PESs are smoother and do not show sudden changes of the slope as is shown, e.g., in the SRP48BOSS results (black) at $0.4 \mathrm{eV}$. In the case of SRP-SCM, the rovibrationally inelastic scattering is significantly larger for all incidence energies.

Figure 6(b) shows the result for the rotationally excited $(\mathrm{v}=0, \mathrm{~J}=11)$ state. Here the crossing point of the elastic and inelastic rovibrationally scattering is the same for SRP-BOSS, SRP48-BOSS, and SRP48-SCM3B while SRP-SCM is different. For the $(v=1, J=6)$ state in Fig. 6(c), we observe the same difference between the SRP-SCM and the others. While the SRP48-BOSS, SRP48-SCM3B, and SRP-BOSS PESs are all similar at high incidence energies, when sampling the corrugation close to the $\mathrm{Cu}(111)$ surface, the SRP-SCM PES is still different between an incidence energy of 0.4 and $0.9 \mathrm{eV}$. Furthermore, the rovibrationally inelastic scattering probability is orders of magnitude higher for very low incidence energies.

The general effect of adding the SCM to both the previously discussed PESs seems to be a broadening in the reaction probability, as well as a larger fraction of rovibrationally inelastic scattering at low energies compared to the BOSS model. The biggest difference between the SRP-SCM and new 
SRP48-SCM3B is that the new SRP48-SCM3B follows the same trend as the BOSS models if the initial rovibrational state is changed, while the SRP-SCM does not. The broadening effect of the SRP-SCM is in general bigger than that of the SRP48-SCM3B.

The differences between the SRP-SCM and SRP48SCM3B PESs of the broadening behaviour are hard to attribute to differences in DFT methods used because the SRP-BOSS PES has the same behaviour as both SRP48 PESs with respect to this behaviour. There are three possible explanations for the difference between the SCMs. First of all, the SRP48SCM3B is fitted to almost 100 times more DFT configurations and also includes displacements of two surface atoms. Second, the coupling potential used in the SRP-SCM had a short cutoff in the $\mathrm{H}-\mathrm{Cu}$ distance that prevented almost all contributions due to second layer displacements. It is known from the work of Bonfanti et al. $^{38}$ that the second layer displacements are very important for the barrier heights. Finally, there are $\mathrm{H}-\mathrm{H}-\mathrm{Cu}$ three-body terms included in the SRP48SCM3B which are, as argued before, not negligible and are not included in the purely additive and pair-potential based SRP-SCM.

\section{E. Comparing with AIMD and experimental results}

When comparing the computed reaction probabilities from the BOSS model and SCM with the results from AIMD ${ }^{9}$ in Figs. 7(a)-7(c), there is a very good agreement across all incidence energies. This was to be expected as the SCM accurately reproduces the DFT used directly by AIMD. Differences are attributed to statistical errors due to the small amount of trajectories in AIMD, the periodicity of the surface displacements in AIMD, the relatively large time step in AIMD, the lack of energy exchange with the lattice, and deviation of the SCM from DFT (42.7 meV RMSE). The reaction probability

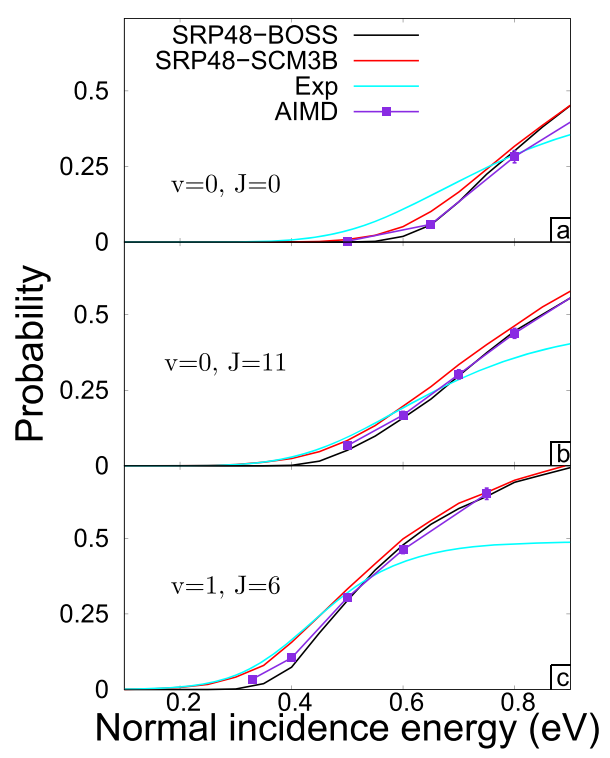

FIG. 7. State-specific reaction probabilities are shown as a function of incidence energy at normal incidence for SRP48-BOSS (black), SRP48-SCM3B (red), and AIMD with SRP48 from Ref. 9 (purple) while a fit to the timeof-flight data of experimental results taken from Ref. 9 are shown in cyan. (a)-(c) show the $(\mathrm{v}=0, \mathrm{~J}=0),(\mathrm{v}=0, \mathrm{~J}=11)$, and $(\mathrm{v}=1, \mathrm{~J}=6)$ rovibrational state-specific reaction probabilities, respectively. curves can be considered to be equivalent, which is extremely useful because it allows to select the correct DFT functional by comparing to experiments at elevated surface temperature using the SCM method. This is orders of magnitude computationally cheaper than AIMD. Figure 8 in Ref. 9 shows that essentially no desorbed molecules with a COM kinetic energy higher than $0.7 \mathrm{eV}$ are measured during the experiment. Therefore, we focus our comparison with the experiment up until an incidence energy of $0.7 \mathrm{eV}$. With that in mind, the rotational and vibrational excited states, shown in Figs. 7(b) and 7(c), respectively, are reproduced very well by the theory, and on the other hand, the rovibrational ground state, in Fig. 7(a), is not. This can be understood due to the $(\mathrm{v}=0$, $\mathrm{J}=0$ ) state being much more sensitive to the shape of the PES around and towards the barrier rather than only the dynamical barrier height as discussed before. Similar to our argument on the accuracy of the SCM fit, we argue here that further improvements in the dynamical model will only lead to a better accuracy when a more accurate DFT functional is found.

\section{F. Initial rovibrational state dependence of the reaction probability}

The computed reaction probabilities have been fitted using a generalized logistics function $(\mathrm{GLF})^{9}$ for all available rovibrational states. There are two important parameters from this fit, the inflection point $\left(E_{0}\right)$ and the width of the curve. The inflection point is where the growth of the reaction probability first starts to decrease, which would be the energy at which the reaction probability is half of its maximum, if the reaction probability would be symmetrical with respect to the inflection point. There is however a small deviation from this symmetry. Note that there are several different definitions of $E_{0}$ in the literature, depending on the chosen fitting function, and that should be considered when comparing $E_{0}$ values from this work. The width is a measure of how broad the reaction probability curve is and it is known to increase when taking into account surface temperature effects. ${ }^{9,13}$ We were not able to compute the uncertainties in the fitted $E_{0}$ and width parameters. Comparison with AIMD and experimental results remains difficult. For AIMD, the limited number of data points in incidence energy, due to the high computational effort, limits the fitting quality of the GLF. On the other hand, the GLF fits to experiments are based on time-of-flight (ToF) measurements of desorption experiments that are subsequently converted to reaction probabilities making use of detailed balance. Here the absolute saturation values are obtained from other molecular beam experiments. The consequences of fitting experimental results in such a way and comparing with theory have recently been discussed. ${ }^{39}$ Figures 8(a) and 8(b) show the fitted $E_{0}$ parameters for $\mathrm{v}=0$ and $\mathrm{v}=1$ at increasing $\mathrm{J}$. For all rovibrational states, the SRP-BOSS and SRP48-BOSS are in very close agreement, even though there is a small difference between the two PESs as discussed above. While the SRP-SCM predicts the same $E_{0}$ as the two BOSS models for J larger than 6, both for $\mathrm{v}=0$ and $\mathrm{v}=1$, and a higher $E_{0}$ at lower $\mathrm{J}$, the SRP48-SCM3B predicts lower $E_{0}$ values except for $\mathrm{v}=1$ and $\mathrm{J}=0$ or $\mathrm{J}=1$. For $\mathrm{v}=0$, the experimental and AIMD results are in reasonable agreement, while for $v=1$, there is a discrepancy between 


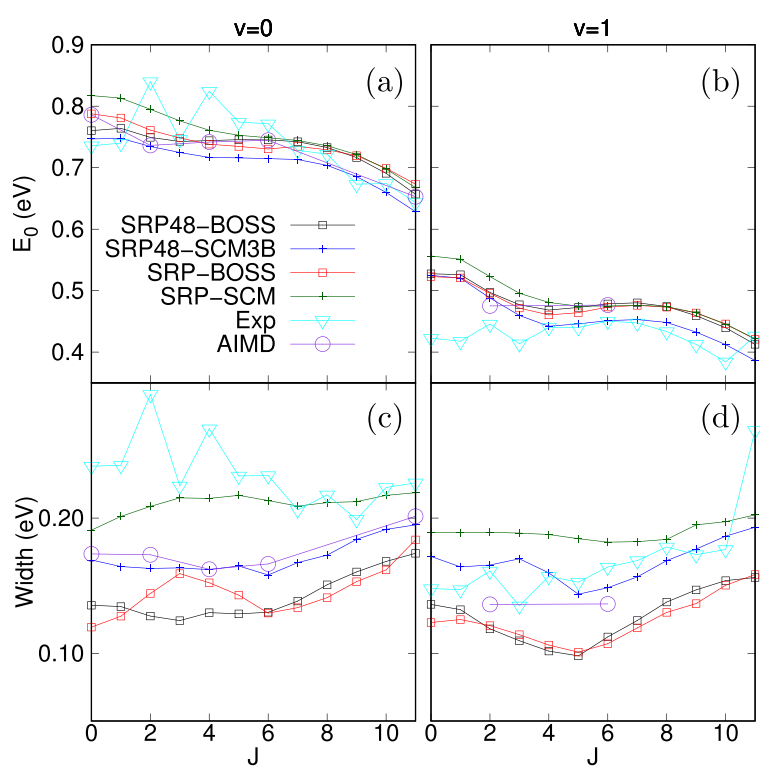

FIG. 8. (a) and (b) show the fitted inflection point $E_{0}$ as a function of the rotational state $\mathrm{J}$ for vibrational state $\mathrm{v}=0$ and $\mathrm{v}=1$, respectively. (c) and (d) show the fitted width as a function of the rotational state $\mathrm{J}$ for vibrational state $\mathrm{v}=0$ and $\mathrm{v}=1$, respectively. The black and blue curves are obtained by fitting state-specific reaction probabilities from this work for SRP48-BOSS and SRP48-SCM3B, respectively. Red and green curves are likewise obtained by refitting the data from Ref. 13 using the GLF. The cyan triangles and purple circles are obtained from Ref. 9 where the experimental (cyan triangles) results are obtained by fitting state-specific time-of-flight data, and the AIMD (purple circles) results are obtained by fitting state-specific reaction probabilities.

experimental and all theoretical results for $\mathrm{J}$ smaller than 3 . It is not clear whether this discrepancy is due to a failure of the theoretical models or because of the way the experimental results have been fitted. On the other hand, the width parameters in Figs. 8(c) and 8(d) show a large dependence on which SCM was used. The width parameters for both SRP-BOSS and SRP48-BOSS are between 0.1 and $0.18 \mathrm{eV}$ and very similar. Including the SCM then increases the width, by approximately $0.05 \mathrm{eV}$ in the case of SRP48 and $0.1 \mathrm{eV}$ in the case of SRP. The AIMD results are in agreement with the increased width of the SRP48-SCM3B, while the SRP-SCM predicts a larger increase in the width. While there is a good agreement between the experimental results and the SRP-SCM for $\mathrm{v}=0$, it is very unlikely that this is due to the quality of the fit, considering the fact that AIMD is not able to reproduce the large widths found in the experiment. Overall, the new SRP48-SCM3B accurately reproduces AIMD results based on the fitted $E_{0}$ and width parameters.

\section{CONCLUSIONS}

A new coupling potential has been fitted for $\mathrm{H}_{2}$ on $\mathrm{Cu}(111)$ within the SCM framework based on the SRP48 density functional using the same setup as AIMD calculations. ${ }^{9}$ A large database of coupling potentials has been constructed for $\mathrm{H}_{2}$ on $\mathrm{Cu}(111)$ at several high symmetry sites for a large amount of surface displacements. Included are configurations with two surface displacements, capturing the $\mathrm{H}-\mathrm{Cu}-\mathrm{Cu}$ threebody interactions which were found to have a negligible non-additivity. In contrast, configurations corresponding to one surface displacements and variable $\mathrm{H}_{2}$ bond distance, describing the $\mathrm{H}-\mathrm{H}-\mathrm{Cu}$ three-body interactions, show a large non-additivity and can therefore not be described accurately using the two-body SCM. The functional form of the coupling potential has thus been extended to have a $\mathrm{H}_{2}$ bond distance dependence of the Rydberg parameters, including $\mathrm{H}-\mathrm{H}-\mathrm{Cu}$ three-body interactions explicitly. Simulations of state-specific desorption experiments using the BOSS model show that the SRP and SRP48 PES are essentially identical, except for the reaction and scattering of rovibrational ground-state $\mathrm{H}_{2}$ even though both functionals were constructed to reproduce the same molecular beam experiments. The differences between the PESs could not be accounted for by the barrier heights, extrinsic curvature of the MEP, or zero point vibrational energy but are instead attributed to the intrinsic curvature of the PES along the MEP. Using the newly developed SCM based on the SRP48, we successfully reproduced AIMD and experimental results and conclude that the SCM can be a good substitute for AIMD in the case of $\mathrm{H}_{2}$ on $\mathrm{Cu}(111)$. For heavier molecules on metal surfaces, where surface motion can be important and the SCM may not be suitable (due to the increased amount of expected energy exchange with the surface), we suggest to include a strain potential to describe the PES of a clean surface using, e.g., embedded atom potentials. ${ }^{40-43}$ Combining this strain potential with the coupling potential yields a full dimensional PES that allows energy exchange with the surface.

\section{ACKNOWLEDGMENTS}

The authors would like to thank Dr. Francesco Nattino for useful discussions. This work was carried out on the National e-infrastructure with the support of SURF Foundation.

\section{APPENDIX: COMPARISON OF MINIMUM ENERGY PATHS OF $\mathrm{H}_{2}$ ON CU(111) FOR SRP AND SRP48}

In Fig. 9(a), the minimum energy paths of both the SRP48 and SRP functionals are shown to be essentially identical. In contrast, the forces along $Z$ and $r$ are slightly different in
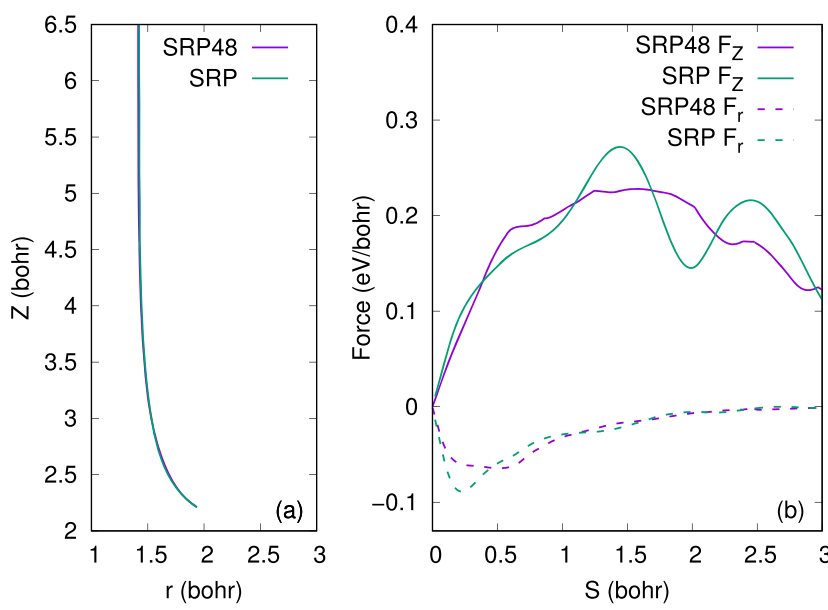

FIG. 9. (a) shows the MEP until the barrier on the bridge site for the SRP48 and SRP PES in purple and green, respectively. (b) shows the forces in Z (solid curves) and $r$ (dashed curves) along the same MEP for SRP48 (purple) and $\mathrm{SRP}$ (green). $\mathrm{S}=0$ bohr corresponds to the barrier position. 
Fig. 9(b). The difference in the force along $Z$ between SRP and SRP48 for large $S$ (far away from the transition state) is not important for the dynamics because there is only a small force along $r$ and thus very little coupling between the two. On the other hand, the small differences at low S (below $1.5 \AA$ ) show that there indeed is a difference in intrinsic curvature around the minimum energy path even though the minimum energy paths are essentially identical.

${ }^{1}$ G. Ertl, Angew. Chem., Int. Ed. 47, 3524 (2008).

${ }^{2}$ T. Zambelli, J. V. Barth, J. Wintterlin, and G. Ertl, Nature 390, 495 (1997).

${ }^{3}$ H. A. Michelsen, C. T. Rettner, and D. J. Auerbach, Surf. Sci. 272, 65 (1992).

${ }^{4}$ H. A. Michelsen, C. T. Rettner, and D. J. Auerbach, Phys. Rev. Lett. 69, 2678 (1992)

${ }^{5}$ C. T. Rettner, D. J. Auerbach, and H. A. Michelsen, Phys. Rev. Lett. 68, 1164 (1992)

${ }^{6}$ C. T. Rettner, H. A. Michelsen, and D. J. Auerbach, Chem. Phys. 175, 157 (1993).

${ }^{7}$ C. T. Rettner, H. A. Michelsen, and D. J. Auerbach, J. Chem. Phys. 102, 4625 (1995).

${ }^{8}$ M. J. Murphy and A. Hodgson, J. Chem. Phys. 108, 4199 (1998).

${ }^{9}$ F. Nattino, A. Genova, M. Guijt, A. S. Muzas, C. Díaz, D. J. Auerbach, and G.-J. Kroes, J. Chem. Phys. 141, 124705 (2014).

${ }^{10}$ G.-J. Kroes, Phys. Chem. Chem. Phys. 14, 14966 (2012).

${ }^{11}$ C. Díaz, E. Pijper, R. A. Olsen, H. F. Busnengo, D. J. Auerbach, and G.-J. Kroes, Science 326, 832 (2009).

${ }^{12}$ D. A. McCormack, G.-J. Kroes, E.-J. Baerends, and R. C. Mowrey, Faraday Discuss. 110, 267 (1998).

${ }^{13}$ M. Wijzenbroek and M. F. Somers, J. Chem. Phys. 137, 054703 (2012).

${ }^{14}$ R. A. Olsen, H. F. Busnengo, A. Salin, M. F. Somers, G.-J. Kroes, and E.-J. Baerends, J. Chem. Phys. 116, 3841 (2002).

${ }^{15}$ C. Díaz, R. A. Olsen, D. J. Auerbach, and G.-J. Kroes, Phys. Chem. Chem. Phys. 12, 6499 (2010).

${ }^{16}$ A. Mondal, M. Wijzenbroek, M. Bonfanti, C. Díaz, and G.-J. Kroes, J. Phys. Chem. A 117, 8770 (2013).

${ }^{17}$ P. Spiering and J. Meyer, J. Phys. Chem. Lett. 9, 1803 (2018).
${ }^{18}$ A. C. Luntz and M. Persson, J. Chem. Phys. 123, 074704 (2005).

${ }^{19}$ K. Shakouri, J. Behler, J. Meyer, and G.-J. Kroes, J. Phys. Chem. Lett. 8, 2131 (2017).

${ }^{20}$ J. Behler and M. Parrinello, Phys. Rev. Lett. 98, 146401 (2007).

${ }^{21}$ B. Kolb, X. Luo, X. Zhou, B. Jiang, and H. Guo, J. Phys. Chem. Lett. 8, 666 (2017).

${ }^{22}$ G. Kresse and J. Hafner, Phys. Rev. B 49, 14251 (1994).

${ }^{23}$ G. Kresse and J. Hafner, J. Phys.: Condens. Matter 6, 8245 (1994).

${ }^{24}$ G. Kresse and J. Furthmüller, Phys. Rev. B 54, 11169 (1996).

${ }^{25}$ D. Vanderbilt, Phys. Rev. B 41, 7892 (1990).

${ }^{26}$ C. C. Marston and G. G. Balint-Kurti, J. Chem. Phys. 91, 3571 (1989).

${ }^{27}$ F. R. Kroeger and C. A. Swenson, J. Appl. Phys. 48, 853 (1977).

${ }^{28}$ K. H. Chae, H. C. Lu, and T. Gustafsson, Phys. Rev. B 54, 14082 (1996).

${ }^{29}$ V. F. Sears and S. A. Shelley, Acta Crystallogr., Sect. A: Found. Crystallogr. 47, 441 (1991).

${ }^{30} \mathrm{~J}$. Stoer and R. Bulirsch, Introduction to Numerical Analysis (Springer New York, New York, NY, 1980).

${ }^{31}$ S. Nave and B. Jackson, J. Chem. Phys. 127, 224702 (2007).

${ }^{32}$ F. Battaglia and F. A. Gianturco, Chem. Phys. 55, 283 (1981).

${ }^{33}$ M. C. van Hemert, J. Chem. Phys. 78, 2345 (1983).

${ }^{34}$ Y. Xiao, W. Dong, and H. F. Busnengo, J. Chem. Phys. 132, 014704 (2010).

${ }^{35}$ F. Nattino, C. Díaz, B. Jackson, and G.-J. Kroes, Phys. Rev. Lett. 108, 236104 (2012).

${ }^{36}$ M. Wijzenbroek and G.-J. Kroes, J. Chem. Phys. 140, 084702 (2014).

${ }^{37}$ R. D. Muino and H. F. Busnengo, Dynamics of Gas-Surface Interactions: Atomic-Level Understanding of Scattering Processes at Surfaces, Springer Series in Surface Sciences (Springer-Verlag, 2013).

${ }^{38}$ M. Bonfanti, C. Díaz, M. F. Somers, and G.-J. Kroes, Phys. Chem. Chem. Phys. 13, 4552 (2011).

${ }^{39}$ S. Kaufmann, Q. Shuai, D. J. Auerbach, D. Schwarzer, and A. M. Wodtke, J. Chem. Phys. 148, 194703 (2018).

${ }^{40}$ S. M. Foiles, M. I. Baskes, and M. S. Daw, Phys. Rev. B 33, 7983 (1986).

${ }^{41}$ Y. Mishin, M. J. Mehl, D. A. Papaconstantopoulos, A. F. Voter, and J. D. Kress, Phys. Rev. B 63, 224106 (2001).

${ }^{42}$ B.-J. Lee, J.-H. Shim, and M. I. Baskes, Phys. Rev. B 68, 144112 (2003).

${ }^{43}$ H. W. Sheng, M. J. Kramer, A. Cadien, T. Fujita, and M. W. Chen, Phys. Rev. B 83, 134118 (2011). 